\title{
Energy-Efficient Binary Power Control with Bit Error Rate Constraint in MIMO-OFDM Wireless Communication Systems
}

\author{
Xi Huang ${ }^{1}$, Xiao-Hu Ge ${ }^{1}$, Yuming Wang ${ }^{1 *}$, Frank $\mathrm{Li}^{2}$ \\ ${ }^{1}$ Dept. Electronics \& Information Engineering, Huazhong University of Science \& Technology, Wuhan, P.R.China. \\ ${ }^{2}$ Department of information and communication technology, Faculty of Engineering and Science University of Agder. \\ ${ }^{*}$ Corresponding author:ymwang@ @ust.edu.cn
}

\begin{abstract}
Motivated by the desire for energy efficiency improvement in information and communication technology (ICT) industry, we explore the idea of optimizing the energy efficiency for MIMO-OFDM wireless communication systems while maintaining user's quality of service $(\mathrm{QoS})$ demand. Based on the binary power control scheme, a power allocation criterion for energy efficiency optimization is derived under a sum power constraint. From a bit error rate (BER) point of view, a protection constraint is configured to guarantee the system QoS. With the aim of energy efficiency optimization under QoS guarantee in MIMO-OFDM wireless communication systems, an energyefficient binary power control with BER constraint (EBPCB) algorithm is proposed based on the power allocation criterion and QoS constraint. Simulations show numerical results of EBPCB in energy efficiency improvement and QoS guarantee.
\end{abstract}

Index Terms-Energy Efficiency; Quality of Service; Power Control; Wireless communication;

\section{INTRODUCTION}

With rapid development of information and communication technologies (ICT), particularly the wireless communication technology, the energy consumption of ICT industry has grown up to $3 \%$ of worldwide energy consumption, which causes about 2\% of worldwide $\mathrm{CO}_{2}$ emissions [1-2]. On the other hand, the increasing energy consumption burdens the electrical bill of network operators. To meet both environmental and economical challenges raised by energy consumption, green wireless communication [3] has been vowed to shift toward energy-efficient designs in all stages of cellular networks while guaranteeing user's QoS.

The green wireless communication explores energy savings of cellular networks in hardware design and manufacture, node deployment, and network operation and management. A holistic approach was proposed for component-, link- and network-level energy savings in cellular networks [4]. For link-level energy savings, significant reduction of energy consumption can be achieved through discontinuous transmission techniques, e.g. sleep modes in base stations [5]. However, to achieve even higher energy efficiency, network-level optimized allocation of wireless resources is a must, especially in power control. Traditional power control mechanisms are designed for voice-centric networks, aiming at a target signalto-noise-and-interference ratio (SINR) for receiving ends [67]. With better exploitation of spatial diversity and reduced transmission power, the adaptive power allocation gains higher achievable SINR by an order of magnitude than the equal power allocation, thus resulting in better coverage [8]. In modern cellular networks, power control has been improved for different optimization targets, such as the spectrum efficiency, average throughput and sum capacity [9-10]. Since energy consumption is closely related to network utilization and life time [11], the network-level energy efficiency is believed to be one of the promising optimization targets. However, further technical questions are brought forward on the proper compromise between energy efficiency and other efficiency objectives, such as the deployment efficiency, spectrum efficiency, delay and QoS [12].

Furthermore, for most power allocation schemes, the acquisition for the perfect centralized knowledge of channel state information (CSI) is a great challenge [13]. To tackle this difficulty, a binary power control (BPC) scheme which leads to a simpler or even distributed solution for performance optimization was proposed [14]. It is demonstrated that the BPC scheme is optimal with respect to the maximal sum rate of a two-cell network. Moreover, extensive simulations suggest that for a greater number of cells, BPC is close to the optimal power allocation with negligible capacity loss [14]. However, the power control algorithm in reference [14] assumes the channel gain as a random variable rather than considering detail influencing factors, such as the pass loss, shadowing and fading effect. Meanwhile, the energy efficiency optimization problem under QoS constraint is not considered in traditional binary power control schemes.

Motivated by the aforementioned gaps, our objective in this paper is to optimize the energy efficiency of Multi-Input Multi-Output and Orthogonal Frequency Division Multiplexing (MIMO-OFDM) wireless communication systems with QoS guarantee. We propose a new algorithm to optimize the energy-efficient power allocation with BER constraint. Our main contributions are summarized as follows:

1) A power allocation criterion for energy efficiency optimization in MIMO-OFDM wireless communication systems is derived under a sum power constraint considering the pass loss, shadowing and fading effect in wireless channels.

2) A new algorithm with BER constraint for user's QoS 
guarantee is proposed to optimize the energy-efficient power allocation in MIMO-OFDM wireless communication systems.

3) The performance of our algorithm is analyzed and some numerical results are presented.

The remainder of this paper is organized as follows. The system model is included in section II. In section III, the energy efficiency optimization problem is formulated with a BER constraint and a sum power constraint and then an optimal criterion is derived for power allocation. Moreover, a new algorithm for energy efficiency optimization with BER constraint is proposed. Simulation results are presented in section IV. Finally, Section V concludes the paper.

\section{SYSTEM MODEL}

In this paper, our research focuses on the downlink performance of wireless communication systems. We investigate the BPC scheme to optimize the energy efficiency of MIMOOFDM wireless communication systems with a BER constraint. A single-cell MIMO-OFDM wireless communication system is illustrated in Fig.1. One base station with $M_{T}$ antennas is located in the center of the cell. Every antenna of the base station is in general assumed to transmit with same power. We set a protection distance $d$ for the base station and assume there are $K$ users uniformly scattering in the $R-d$ circular disk around the base station. Each user is integrated with $M_{R}$ antennas.

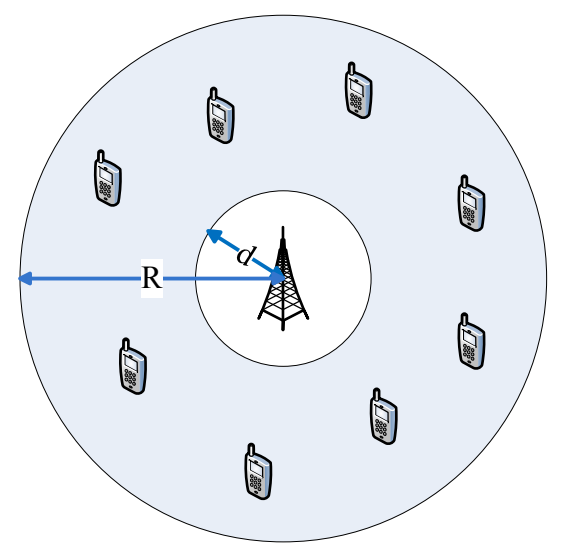

Fig. 1. System model of MIMO-OFDM wireless communication systems.

To simplify the modeling complexity of the OFDM scheme, all orthogonal $N$ subcarriers are regrouped into $N$ subchannels by the OFDM scheme. For one moment, without loss of generality, there are $N$ subchannels enabled for data transmission. Each signal in subchannels experiences independent path loss, shadowing effect and the multi-path fading. Moreover, interference from other users is ignored in this single cell.

According to the assumptions above, the received signal power $S_{i}$ in subchannel $i$ is given by

$$
S_{i}=\frac{\omega z_{i}^{2}}{R_{i}^{\sigma_{r}}} P_{i}
$$

where $R_{i}$ is the distance between the base station and user i. $P_{i}$ is the transmission power over subchannel $i . \omega$ is the lognormal shadowing coefficient, $z_{i}^{2}$ is the Reyleigh fading coefficient of subchannel $i$ and $\sigma_{r}$ is the path loss coefficient.

In this paper, the energy efficiency in wireless communication systems is defined by the ratio of system capacity to the total system transmission power. Assuming the maximum achievable channel capacity is Shannon capacity, the energy efficiency of wireless communication systems is derived as follows

$$
\eta=\frac{\sum_{i=1}^{N} \log _{2}\left(1+\frac{S_{i}}{n_{0}}\right)}{P_{\text {total }}}
$$

where $\eta$ is the energy efficiency of wireless communication systems. $n_{0}$ is the additive white Gaussion noise (AWGN) in wireless subchannels. $P_{\text {total }}$ is the total system transmission power.

In wireless communication systems, the bit error rate depends on the modulation scheme applied. In this paper, we adopt BDPSK modulation to investigate the performance of BER in subchannels. The BER $P_{B E R}$ with BDPSK modulation is expressed by

$$
P_{B E R}=\frac{1}{2} e^{-\frac{\varepsilon_{b}}{N_{0}}}
$$

where $\varepsilon_{b}$ is the bit energy and $N_{0}$ is the noise power spectrum density.

To evaluate the BER performance of wireless communication systems, we define the system average bit error rate as follows

$$
P_{\text {aver_BER }}=\frac{\sum_{i=1}^{N} P_{B E R i}}{N}
$$

where $P_{\text {aver }} B E R$ is the system average bit error rate. $P_{B E R i}$ is the bit error rate of the received signal in subchannel $i$.

\section{ENERGY EFFICIENCY OPTIMIZATION WITH BER CONSTRAINT}

Based on the models described above, we adopt the BPC scheme to allocate the transmission power for subchannels to optimize the system energy efficiency while maintaining a given QoS demand.

\section{A. Problem formulation}

Firstly, the set of subchannels enabled for transmission is denoted as $C$ which is expressed as

$$
C H_{i} \in C, C=\left\{C H_{i} \mid 1 \leq i \leq N\right\}
$$

where $C H_{i}$ is the subchannel $i$.

Since the value of transmission power for subchannels is fed from a binary feasible set, we divide the subchannel set $C$ into two subsets: one is the maximum power transmission subchannel subset $K_{p \max }^{M}$ in which the subchannel transmission power is $P_{\max }$; the other is the minimum power transmission subchannel subset $K_{p \min }^{N-M}$ in which the subchannel transmission power is $P_{\min }$. Moreover, we assume the number 
of subchannels in $K_{p \max }^{M}$ is $M$. The total transmission power of $K_{p \max }^{M}$ is denoted as $P_{\max \_t o t a l}$ and the total transmission power of $K_{p \min }^{N-M}$ is denoted as $P_{\min \_t o t a l}$. In this case, the relationship of $P_{\text {total }}, P_{\max \_t o t a l}$ and $\bar{P}_{\text {min_total }}$ is described as follows

$$
\left\{\begin{array}{l}
P_{\text {total }}=P_{\max \text { total }}+P_{\min \text { total }} \\
P_{\max \text { total }}=M \times P_{\max } \\
P_{\min \text { total }}=(N-M) \times P_{\min }
\end{array}\right.
$$

To search for a system-wide optimization of the system energy efficiency, a sum power constraint $P_{\text {total }}$ is set and $P_{\text {max } t \text { total }}$ in the maximum power transmission subchannel subset is assumed to be fixed as a constant.

In practical wireless communication systems, the QoS at user end directly depends on the BER performance. For QoS guarantees, we investigate the optimal solution in energy efficiency subject to a BER constraint as well as a sum power constraint described above in the following parts. Then, the whole optimization problem is summarized as follows, where the BER upper bound is denoted as $\tilde{b}$.

$$
\max \quad \eta=\frac{\sum_{i=1}^{M} \log _{2}\left(1+\frac{S_{i}}{n_{0}}\right)}{P_{\max \text { total }}}
$$

Subject to

Constraint 1: $P_{\text {total }}$ and $P_{\max \_ \text {total }}$ is fixed as a constant. Constraint 2: $P_{\text {aver_BER }} \leq \tilde{b}$.

\section{B. Energy efficiency optimal solution}

The core idea of the system energy efficiency optimization lies in that the power allocation based on the BPC scheme should maximize the system energy efficiency. Applying this idea to power allocation, a candidate wireless subchannel $\mathrm{CH}_{k}$ is assigned into $K_{p \max }^{M}$ only when the energy efficiency of $K_{p \max }^{M}$ including $\mathrm{CH}_{k}$ is no less than the energy efficiency of $K_{p \max }^{M}$ without $\mathrm{CH}_{k}$, otherwise $\mathrm{CH}_{k}$ should be assigned into $K_{p \min }^{N-M}$.

Based on constraint 1, the transmission power of each subchannel in $K_{p \max }^{M}$ when subchannel $C H_{k}$ is assigned into $K_{p \max }^{M}$ is derived as follows

$$
P_{\text {max } \_1}=\frac{P_{\text {max } \_ \text {total }}}{M}
$$

Similarly, the transmission power of each subchannel in $K_{p \max }^{M}$ when subchannel $C H_{k}$ is not assigned into $K_{p \max }^{M}$ is given by

$$
P_{\max \_2}=\frac{P_{\text {max } \_ \text {total }}}{M-1}
$$

Based on the system energy efficiency model described in section II, the energy efficiency $\eta_{i \in N}^{a}$ of $K_{p \max }^{M}$ including subchannel $\mathrm{CH}_{k}$ is denoted as

$$
\eta_{i \in N}^{a}=\frac{\sum_{i=1}^{\mathrm{M}} \log _{2}\left(1+\frac{\frac{\omega z_{i}^{2}}{R_{i}{ }^{\sigma_{r}}} P_{\max }{ }^{1}}{n_{0}}\right)}{P_{\max \text { total }}}
$$

the energy efficiency $\eta_{i \in N, i \neq k}^{b}$ of $K_{p \max }^{M}$ without subchannel $C H_{k}$ is denoted as

$$
\eta_{i \in N, i \neq k}^{b}=\frac{\sum_{i=1, i \neq k}^{\mathrm{M}-1} \log _{2}\left(1+\frac{\frac{\omega z_{i}^{2}}{R_{i}{ }^{\sigma} r} P_{\mathrm{max}} \_^{2}}{n_{0}}\right)}{P_{\max \text { total }}}
$$

According to the core idea of the system energy efficiency optimization described above, only satisfying the condition $\eta_{i \in N}^{a} \geq \eta_{i \in N, i \neq k}^{b}$ can the candidate wireless subchannel $\mathrm{CH}_{k}$ be finally assigned into $K_{p \max }^{M}$. This condition is expressed by

$$
\frac{\sum_{i=1}^{\mathrm{M}} \log _{2}\left(1+\frac{\frac{\omega z_{i}^{2}}{R_{i} \sigma_{r}} P_{\max \_} \__{0}}{n_{0}}\right)}{P_{\max \text { total }}} \geq \frac{\sum_{i=1, i \neq k}^{\mathrm{M}-1} \log _{2}\left(1+\frac{\frac{\omega z_{i}^{2}}{R_{i} \sigma_{r}} P_{\max } \_^{2}}{n_{0}}\right)}{P_{\max \text { total }}}
$$

From appendix of (12), the optimal power allocation criterion for system energy efficiency is derived as follows

$$
\frac{\frac{\omega z_{k}^{2}}{R_{k} \sigma_{r}} P_{\max \_2}}{n_{0}} \geq\left(\frac{M}{M-1}\right)^{M-1}-1
$$

To simplify the expression (13), we set it as follows

$$
S N R_{k} \geq \gamma
$$

where the left side of expression (14) is the signal to noise ratio (SNR) of the candidate wireless subchannel $\mathrm{CH}_{k}$, which is denoted as $S N R_{k}$. The right side of expression (14) is the power allocation threshold value, which is denoted as $\gamma$.

Based on (14), the system energy efficiency optimization can be carried out by comparing the $S N R_{k}$ of the candidate subchannel $\mathrm{CH}_{k}$ with the threshold value $\gamma$. If the comparison result satisfies the expression (14), the candidate subchannel $\mathrm{CH}_{k}$ is assigned into the maximum power transmission subchannel subset $K_{p \max }^{M}$. Otherwise, $C H_{k}$ is assigned into the minimum power transmission subchannel subset $K_{p \min }^{N-M}$.

\section{Algorithm design}

Based on constraint $\mathbf{1}$ and 2, an energy-efficient binary power control with bit error rate constraint (EBPCB) algorithm is designed for energy efficiency optimization in MIMO-OFDM wireless communication systems while maintaining the system QoS. Firstly, all subchannels of wireless subchannel set $C$ are degressively ordered according to the CSI. Then the process of power allocation optimization with BER constraint begins. The key idea of the power allocation optimization is to assign a candidate wireless subchannel $\mathrm{CH}_{k}$ into the maximum power transmission subchannel subset $K_{p \max }^{M}$, then calculate the system average BER and $S N R_{k}$. If the calculation results satisfy constraint 2 and the power allocation criterion, the candidate wireless subchannel $\mathrm{CH}_{k}$ is finally added to $K_{p \max }^{M}$. Otherwise, $\mathrm{CH}_{k}$ is assigned into the minimum power transmission subchannel subset $K_{p \min }^{N-M}$. The detailed EBPCB algorithm is illustrated in Algorithm 1:

ALGORITHM 1: Energy-efficient binary power control 
Input: $P_{\text {total }}, P_{\text {max }}$ total,$\gamma$

Output: $P_{\max }, P_{\min }, K_{p \max }^{M}, K_{p \min }^{N-M}$

Initialization: Create a wireless sub-channel set $C$ with $N$ subchannels, the maximum power transmission subchannel subset $K_{p \max }^{M}$ and the minimum power transmission subchannel subset $K_{p \min }^{N-M}$.

$$
\begin{aligned}
& C=\left\{C H_{i} \mid 1 \leq i \leq N\right\}, \\
& K_{p \max }^{M}=\phi, \\
& K_{p \min }^{N-M}=\phi .
\end{aligned}
$$

\section{Begin:}

1) Create a new set $\tilde{C}$ from the set $C$ by a descending order of $\frac{\omega z_{i}^{2}}{R_{i}{ }^{\sigma_{r}}}$

$$
\tilde{C}=\left\{C H_{i} \mid \forall(1 \leq i \leq k \leq N), \frac{\omega z_{i}^{2}}{R_{i}{ }^{\sigma_{r}}} \geq \frac{\omega z_{k}^{2}}{R_{k}{ }^{\sigma_{r}}}\right\} .
$$

2) for $i=1: N$ do

$$
\begin{gathered}
P_{\max }=\frac{P_{\max \_ \text {total }}}{i-1}, \\
S N R_{i}=\frac{\frac{\omega z_{i}^{2}}{R_{i}{ }_{r}} P_{\max }}{n_{0}}, \\
P_{B E R i}=\frac{1}{2} e^{-\frac{\varepsilon_{b i}}{N_{0}}}, \\
P_{\text {aver_BER }}=\frac{\operatorname{sum}\left(P_{B E R 1}: P_{B E R i}\right)}{i} . \\
\text { if } P_{\text {aver_ } B E R}>\tilde{b} \\
M=i-1, \\
\text { break } \\
\text { else if } S N R_{i}<\gamma
\end{gathered}
$$

$$
\begin{aligned}
& \text { M } M=i-1, \\
& \text { break } \\
& \text { end if } \\
& \text { end if }
\end{aligned}
$$

end for

3) add $C H_{j}(1 \leq j \leq M)$ into $K_{p \max }^{M}$, add $C H_{j}(M+1 \leq j \leq N)$ into $K_{p \min }^{N-M}$, $P_{\max }=\frac{P_{\max }{ }^{\text {total }}}{M}, P_{\min }=\frac{P_{\text {total }}-P_{\max t o t a l}}{N-M}$.

\section{end Begin}

\section{SIMULATION RESULTS AND PERFORMANCE ANALYSIS}

Based on the system models described in section II, we will now measure the proposed EBPCB algorithm performance in MIMO-OFDM wireless communication systems through Monte Carlo simulations. To evaluate the performance distinctly, we compare the performance of EBPCB algorithm with two other power control schemes: the binary power control scheme without optimization aim (BPC) and the energyefficient binary power control scheme without BER constraint (EBPC).

In our simulation, we assume users are uniformly distributed in a circular disk around the base station since we set an protection distance $d$ for the base station. The radius of the single-cell is ranged from 300 to $500 \mathrm{~m}$ and the protection distance $d$ is assumed as $50 \mathrm{~m}$. Further simulation details are configured as follows: the system bandwidth is assumed as 1 Mhz; the bit rate is assumed as $10 \mathrm{~kb} / \mathrm{s}$ in all subchannels for simplicity; the BER upper bound is configured as 10E-13 [15]; the total transmission power of the base station is ranged from 0.6 to 1.4 watt $(\mathrm{W})$; the pass loss coefficient is ranged from 3.8 to 4.1 ; Considering the OFDM scheme used in MIMO wireless communication systems, the number of subchannles is ranged from 8 to 128 ; the AWGN $n_{0}$ is configured as $0.1 \mathrm{~W}$.

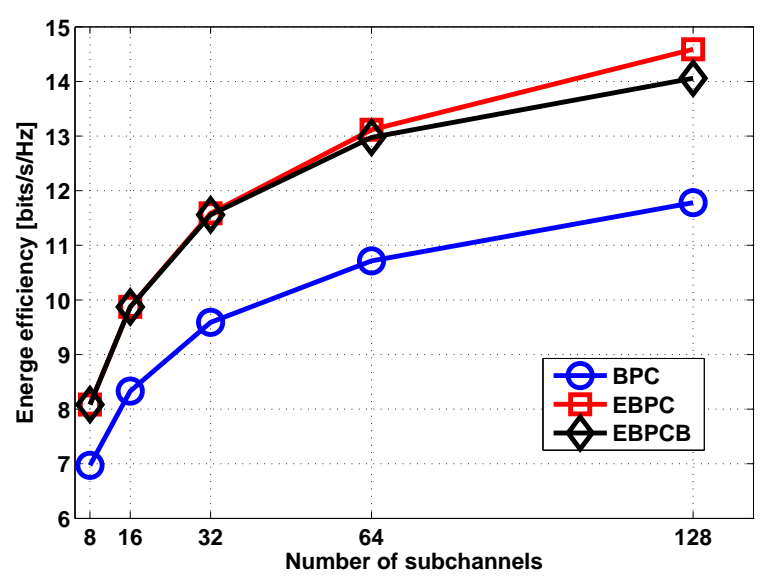

Fig. 2. Comparison energy efficiency of EBPCB, BPC and EBPC with different number of subchannels.

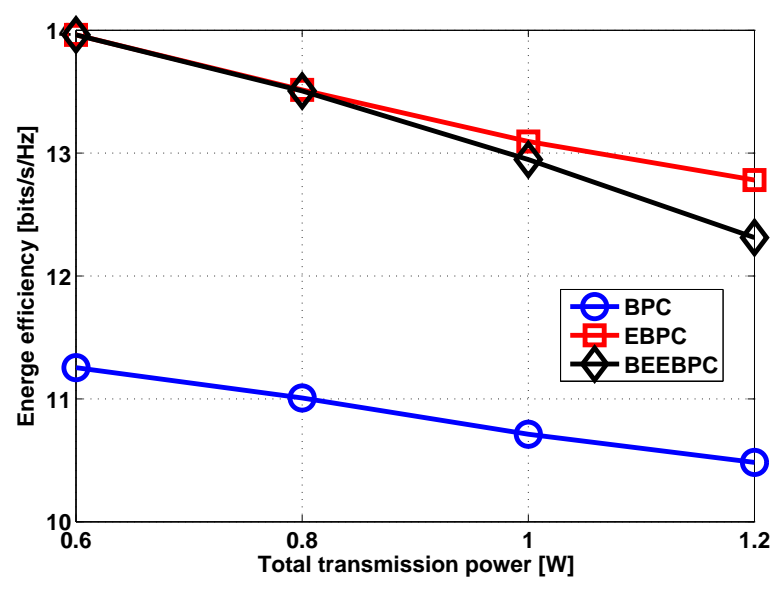

Fig. 3. Comparison energy efficiency of EBPCB, BPC and EBPC with different total transmission power.

Fig.2 shows the energy efficiency comparison of EBPCB, $\mathrm{BPC}$ and EBPC as a function of number of subchannels. From 
Fig.2, it is clearly seen that EBPCB and EBPC outperform the BPC scheme in energy efficiency. This result demonstrates the effect of energy efficiency optimization by optimal power allocation. In terms of EBPCB and EBPC, the curves show that the energy efficiency of the two schemes is approximately the same when the number of subchannels is less than 32 . Nonetheless, when the number of subchannels is larger than 32, EBPCB yields a marginal loss in energy efficiency as compared with EBPC. Moreover, the energy efficiency of the three schemes increases with the number of subchannels.

Fig. 3 shows the energy efficiency comparison of EBPCB, $\mathrm{BPC}$ and EBPC as a function of total transmission power. From Fig.3, it is clearly seen that EBPCB and EBPC outperform the BPC scheme in energy efficiency. This result demonstrates the effect of energy efficiency optimization by optimal power allocation. In terms of EBPCB and EBPC, the curves show that the energy efficiency of the two schemes is approximately the same when the total transmission power is less than $0.8 \mathrm{~W}$. Nonetheless, when the number of subchannels is larger than $0.8 \mathrm{~W}$, EBPCB yields a marginal loss in energy efficiency as compared with EBPC. Moreover, the energy efficiency of the three schemes decreases with the total transmission power.

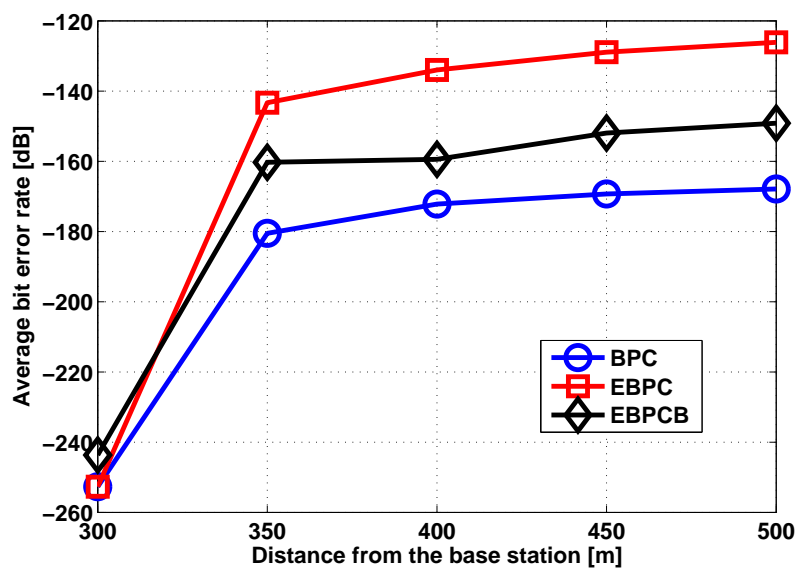

Fig. 4. Comparison average bit error rate of EBPCB, BPC and EBPC with different distance from the base station.

Fig.4 shows the average bit error rate comparison of EBPCB, BPC and EBPC as a function of distance from the base station. From Fig.4, it is remarked that the BPC scheme outperforms EBPC and EBPCB in average bit error rate. This result demonstrates the fact that there is a fundamental trade-off between energy efficiency optimization and the BER performance. In terms of EBPCB and EBPC, it is noted that EBPC yields better performance in average bit error rate when the distance from the base station is less than $320 \mathrm{~m}$. Nonetheless, EBPCB outperforms EBPC in average bit error rate when the distance from the base station is larger than $320 \mathrm{~m}$. It is notable that the average bit error rate of EBPCB is always below $-130 \mathrm{~dB}$ due to the bit error rate constraint 2. Moreover, the average bit error rate of the three schemes

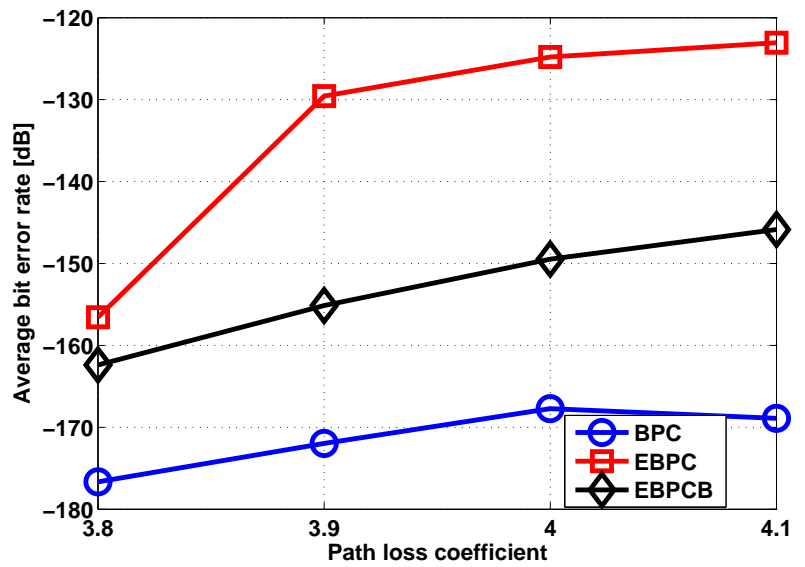

Fig. 5. Comparison average bit error rate of EBPCB, BPC and EBPC with different path loss coefficient.

increases with the distance from the base station.

Fig.5 shows the average bit error rate comparison of EBPCB, BPC and EBPC as a function of path loss coefficient. From Fig.5, it is remarked that the BPC scheme outperforms EBPC and EBPCB in average bit error rate. This result demonstrates the fact that there is a fundamental trade-off between energy efficiency optimization and the BER performance. In terms of EBPCB and EBPC, it is noted that EBPCB significantly outperforms EBPC in average bit error rate. It is notable that the average bit error rate of EBPCB is always below $-130 \mathrm{~dB}$ due to the bit error rate constraint 2 . Moreover, the general trend of the average bit error rate of the three schemes is ascending with the path loss coefficient.

\section{CONCLUSION}

In this paper, we explore the idea of optimizing the energy efficiency for MIMO-OFDM wireless communication systems while maintaining a given QoS demand. Assuming the perfect CSI is presented in each subchannel, we adopt the BPC scheme for energy efficiency optimization and a criterion for power allocation is derived under a sum power constraint. To guarantee the system QoS, a protection constraint is configured from a BER point of view. Base on the power allocation criterion and QoS constraint, a EBPCB algorithm is proposed with energy efficiency optimization and QoS guarantee in MIMO-OFDM wireless communication systems. Comparison of EBPCB, BPC and EBPC schemes in simulation results shows the new EBPCB algorithm has a good performance in energy efficiency with a given guarantee in the system QoS.

\section{ACKNOWLEDGMENT}

$\mathrm{X}$. Huang and $\mathrm{X}$. Ge would like to acknowledge the support from the RCUK for the UK-China Science Bridges Project: R\&D on (B) $4 \mathrm{G}$ Wireless Mobile Communications. X. Huang, X. Ge and F.Y. Li acknowledge the support from the EU FP7-PEOPLE-IRSES program, project acronym S2EuNet (Grant no.: 247083). X. Huang and X. Ge also acknowledge the support from the National Natural Science Foundation of China (NSFC) 
(Grant No.: 60872007), National 863 High Technology Program of China (Grant No.: 2009AA01Z239) and the Ministry of Science and Technology (MOST), China, International Science and Technology Collaboration Program (Grant No.: 0903), and Hubei Provincial Science and Technology Department (Grant No.: 2011BFA004).

\section{APPENDIX OF (12)}

In this appendix, the optimal power allocation criterion for system energy efficiency is derived. Based on the core idea of the system energy efficiency optimization, the condition for the candidate subchannel $C H_{k}$ to be assigned into $K_{p \max }^{M}$ is expressed as follows

$$
\begin{gathered}
\frac{\sum_{i=1}^{\mathrm{M}} \log _{2}\left(1+\frac{\frac{\omega z_{i}^{2}}{R_{i}{ }^{\sigma_{r}}} P_{\max } \__{1}}{n_{0}}\right)}{P_{\max \text { total }}} \geq \frac{\sum_{i=1, i \neq k}^{\mathrm{M}-1} \log _{2}\left(1+\frac{\frac{\omega z_{i}^{2}}{R_{i} \sigma_{r}} P_{\max } \_^{2}}{n_{0}}\right)}{P_{\max \text { total }}} \\
\prod_{i=1}^{M}\left(1+\frac{\frac{\omega z_{i}^{2}}{R_{i}{ }^{\sigma_{r}}} P_{\max \_1}}{n_{0}}\right) \geq \prod_{i=1, i \neq k}^{M-1}\left(1+\frac{\frac{\omega z_{i}^{2}}{R_{i} \sigma_{r}} P_{\max \_2}}{n_{0}}\right)
\end{gathered}
$$

Considering (8) and (9), we can derive the following expression

$$
P_{\max \_1} \leq P_{\max \_2}
$$

Based on (17), (16) is further derived as follows

$$
\begin{aligned}
& \left(1+\frac{\frac{\omega z_{i}^{2}}{R_{i} \sigma_{r}} P_{\max \_2}}{n_{0}}\right) \prod_{i=1, i \neq k}^{M-1}\left(1+\frac{\frac{\omega z_{i}^{2}}{R_{i} \sigma_{r}} P_{\max \_1}}{n_{0}}\right) \geq \\
& \prod_{i=1}^{M}\left(1+\frac{\frac{\omega z_{i}^{2}}{R_{i} \sigma_{r}} P_{\max \_1}}{n_{0}}\right) \geq \prod_{i=1, i \neq k}^{M-1}\left(1+\frac{\frac{\omega z_{i}^{2}}{R_{i}{ }^{\prime}} P_{\max \_2}}{n_{0}}\right) \\
& \Downarrow \\
& 1+\frac{\frac{\omega z_{i}^{2}}{R_{i} \sigma_{r}} P_{\max \_2}}{n_{0}} \geq \frac{\prod_{i=1, i \neq k}^{M-1}\left(1+\frac{\frac{\omega z_{i}^{2}}{R_{i}{ }^{\sigma}} P_{\max } \_^{2}}{n_{0}}\right)}{\prod_{i=1, i \neq k}^{M-1}\left(1+\frac{\omega z_{i}^{2}}{R_{i}{ }^{\sigma} P_{\max }{ }^{1}}\right)} \\
& \Downarrow \\
& 1+\frac{\frac{\omega z_{i}^{2}}{R_{i}{ }^{\sigma r}} P_{\max \_2}}{n_{0}} \geq \frac{\prod_{i=1, i \neq k}^{M-1}\left(n_{0}+\frac{\omega z_{i}^{2}}{R_{i}{ }^{\sigma r}} P_{\max \_2}\right)}{\prod_{i=1, i \neq k}^{M-1}\left(n_{0}+\frac{\omega z_{i}^{2}}{R_{i}{ }^{\sigma r}} P_{\max \_1}\right)}
\end{aligned}
$$

Since the AWGN $n_{0}$ is obviously less than the received signal in subchannel $i$, we can approximate (20) as follows

$$
1+\frac{\frac{\omega z_{i}^{2}}{R_{i}{ }^{\sigma_{r}}} P_{\max \_2}}{n_{0}} \geq \frac{\prod_{i=1, i \neq k}^{M-1} \frac{\omega z_{i}^{2}}{R_{i}{ }^{\sigma_{r}}} P_{\max \_2}}{\prod_{i=1, i \neq k}^{M-1} \frac{\omega z_{i}^{2}}{R_{i}{ }^{\sigma_{r}}} P_{\max \_1}}
$$

$$
\begin{aligned}
& 1+\frac{\frac{\omega z_{i}^{2}}{R_{i}{ }^{\sigma_{r}}} P_{\max \_2}}{n_{0}} \geq \frac{\prod_{i=1, i \neq k}^{M-1} P_{\max \_2}}{\prod_{i=1, i \neq k}^{M-1} P_{\max \_1}} \\
& \Downarrow \\
& \frac{\frac{\omega z_{k}^{2}}{R_{k} \sigma_{r}} P_{\max \_} 2}{n_{0}} \geq\left(\frac{M}{M-1}\right)^{M-1}-1
\end{aligned}
$$

This completes the derivation.

\section{REFERENCES}

[1] I. Humar, X. Ge, X. Lin, M. Jo and M. Chen, "Rethinking energyefficiency models of cellular networks with embodied energy," IEEE Network Magazine, vol. 25, no. 2, pp. 40-49, March 2011.

[2] A. Fehske, G. Fettweis, J. Malmodin and G. Biczok, "The Global Footprint of Mobile Communications: The Ecological and Economic Perspective," IEEE Communications Magazine, vol. 49, no. 8, pp. 5562, Aug. 2011.

[3] Y. Chen, S. Zhang and S. Xu, "Fundamental Trade-offs on Green Wireless Networks," IEEE Communications Magazine, vol. 49, no. 6, pp. 30-37, June 2011.

[4] L. M. Correia, D. Zeller, O. Blume, D. Ferling and Y. Jading, "Challenges and Enabling Technologies for Energy Aware Mobile Radio Networks," IEEE Communications Magazine, vol. 48, no. 11, pp. 66-72, Nov. 2010.

[5] I. Ashraf, F. Boccardi and L. Ho, "SLEEP Mode Techniques for Small Cell Deployments," IEEE Communications Magazine, vol. 49, no. 8, pp. 72-79, Aug. 2011.

[6] J. Zander, "Performance of optimum transmitter power control in cellular radio systems," IEEE Trans. Veh. Technol., vol.41, no. 1, pp.57-62, Feb. 1992.

[7] G. J. Foschini, Z. Miljanic, "A simple distributed autonomous power control algorithm and its convergence," IEEE Trans. Veh. Technol.,vol. 42, no. 4, pp. 641-646, Nov. 1993.

[8] R. Chen, J. G. Andrews, R. W. Heath and A. Ghosh, "Power Control for Cellular MIMO Systems," in Proc. IEEE Global Telecommunications Conference, 2006 (GLOBECOM '06), pp. 1-5, Nov. 2006.

[9] Y. Cho, M. Kim and F. A. Tobagi, "Adaptive Throughput Optimization for MIMO Systems in Rayleigh Fading Channels," in Proc. IEEE International Conference on Communications, 2007 (ICC '07), pp. 2574-2579, June 2007.

[10] E. Vagenas, G. S. Paschos and S. A. Kotsopoulos, "Beamforming Capacity Optimization for MISO Systems with Both Mean and Covariance Feedback," IEEE Trans. on Wireless Communications, vol. 10, no. 9, pp. 2994-3001, Sep. 2011

[11] J. Feng, C. Chang, S. Sayilir, Y. Lu, B. Jung, D. Peroulis and Y. C. $\mathrm{Hu}$, "Energy-Efficient Transmission for Beamforming in Wireless Sensor Networks," in Proc. IEEE Communication Society Conference on Sensor Mesh and Ad Hoc Communication and Networks, 2010 (SECON), pp.1-9, June 2010.

[12] H. Bogucka, A. Conti, "Degrees of Freedom for Energy Savings in Practical Adaptive Wireless Systems," IEEE Communications Magazine, vol. 49, no. 6, pp. 38-45, June 2011.

[13] B. Zayen, M. Haddad, A. Hayar and G. E. Oien "Binary power allocation for cognitive radio networks with centralized and distributed user selection strategies" Physical Communication Journal, vol.1, no.3, pp.183-193, Sep.2008.

[14] S. G. Kiani, G. E. Oien and D. Gesbert, "Maximizing Multicell Capacity Using Distributed Power Allocation and Scheduling," in Proc. IEEE Wireless Communications and Networking Conference, 2007 (WCNC 2007), pp.1690-1694, March 2007.

[15] Y. Zhao, S. G. Haggman, "BER Analysis of OFDM Communication Systems with Intercarrier Interference," in Proc. International Conference on Communication Technology, 1998 (ICCT '98), pp. 1-5, Oct. 1998. 